\section{(1)}

CrossMark

\title{
Oral iron supplementation with ferric maltol in patients with pulmonary hypertension
}

To the Editor:

In patients with pulmonary hypertension and iron deficiency anaemia, oral iron supplementation with ferric maltol was well tolerated and restored iron and haemoglobin.

Iron deficiency is common in patients with pulmonary hypertension [1, 2]. The underlying pathomechanisms are complex and include impaired iron absorption, elevated hepcidin levels, augmented gastrointestinal loss and reduced availability from the reticuloendothelial system $[1,3,4]$. In patients with pulmonary hypertension, iron deficiency is associated with aggravated symptoms and an increased mortality risk $[3,5]$.

Currently available oral iron formulations such as ferrous $\left(\mathrm{Fe}^{2+}\right)$ sulfate, ferrous gluconate and ferrous fumarate are often poorly tolerated. In addition, drug efficacy in terms of iron restoration and haemoglobin regeneration may be impaired in patients with pulmonary hypertension [2]. For these reasons, current guidelines, while acknowledging the need for further data, recommend intravenous iron supplementation in patients with pulmonary hypertension and iron deficiency [6]. With newer intravenous iron formulations such as ferric carboxymaltose, risk of serious reactions appears to be very low [7], but intravenous iron therapy is nonetheless not entirely free of risk and is more cumbersome than oral therapy.

Ferric maltol is an orally available formulation of ferric $\left(\mathrm{Fe}^{3+}\right)$ iron and maltol, a naturally occurring sugar derivative [8] that has been shown to be safe, effective and well tolerated in patients with inflammatory bowel disease, including patients who were previously intolerant to other oral iron formulations [9]. Ferric maltol is approved in the United States and Europe for treatment of iron deficiency in adults, but it is unknown whether this compound is an effective remedy against iron deficiency anaemia in patients with pulmonary hypertension.

Here, we present the results of the Oral Iron Supplementation with Ferric Maltol in Patients with Pulmonary Hypertension (ORION-PH) study, an explorative, open-label, single-centre study assessing preliminary safety, tolerability and efficacy of ferric maltol in patients with various forms of pulmonary hypertension and iron deficiency anaemia (clinical trials.gov, NCT03371173). The study enrolled patients between May 2018 and Nov 2019. Ferric maltol was administered at the approved dose of $30 \mathrm{mg}$ twice daily. The primary study objective was the change in haemoglobin levels from baseline to week 12 of treatment with ferric maltol. Secondary objectives included the effects of oral ferric maltol on iron status, World Health Organization functional class, 6-min walk distance (6MWD), serum levels of the N-terminal pro-brain natriuretic peptide (NT-proBNP), and echocardiography parameters of right ventricular (RV) function. Per protocol, changes from baseline to week 12 were analysed in patients who completed the 12 -week study period. Statistical comparisons of baseline and week 12 data were performed with Chi-squared tests or paired Wilcoxon tests, as appropriate. Safety and tolerability were assessed in all study participants at week 6 and week 12, and 4 weeks after the last intake of study medication. The study was approved by the local ethics committee, and all patients provided written informed consent.

@ERSpublications

Oral ferric maltol was well tolerated and effective in patients with pulmonary hypertension and iron deficiency anaemia. Restoration of iron stores corrected anaemia and resulted in improved right ventricular function and exercise tolerance https://bit.ly/2zMwvxc

Cite this article as: Olsson KM, Fuge J, Brod T, et al. Oral iron supplementation with ferric maltol in patients with pulmonary hypertension. Eur Respir J 2020; 56: 2000616 [https://doi.org/10.1183/ 13993003.00616-2020]. 
Main inclusion criteria were a diagnosis of any form of pulmonary hypertension confirmed by a (historical) right heart catheter showing a mean pulmonary artery pressure $\geqslant 25 \mathrm{mmHg}$ at rest and stable pulmonary hypertension medication for $\geqslant 3$ months, the presence of mild-to-moderate iron deficiency anaemia as defined by a haemoglobin concentration $\geqslant 7 \mathrm{~g} \cdot \mathrm{dL}^{-1}$ and $<12 \mathrm{~g} \cdot \mathrm{dL}^{-1}$ in females or $\geqslant 8 \mathrm{~g} \cdot \mathrm{dL}^{-1}$ and $<13 \mathrm{~g} \cdot \mathrm{dL}^{-1}$ in males, and serum ferritin $<100 \mu \mathrm{g} \cdot \mathrm{L}^{-1}$, or $100-300 \mu \mathrm{g} \cdot \mathrm{L}^{-1}$ and transferrin saturation $<20 \%$ at screening. Main exclusion criteria were other active haematological disorders, malignancy, active bleeding, ongoing oral or intravenous iron supplementation, concomitant erythropoietin treatment and severe kidney disease with a glomerular filtration rate $<30 \mathrm{~mL} \cdot \mathrm{min}^{-1}$.

The study was originally designed to enrol 25 patients, but was closed after inclusion of 22 patients due to slow recruitment. The median (range) age of the enrolled patients was 57 (49-71) years; 82\% were female. Underlying conditions were pulmonary arterial hypertension $(n=14,64 \%)$, pulmonary hypertension due to left heart disease $(n=1,5 \%)$ and inoperable chronic thromboembolic pulmonary hypertension $(n=7,32 \%)$. Haemodynamics at the time of diagnosis showed severe pre-capillary pulmonary hypertension with mean \pm SD pulmonary artery pressure of $50 \pm 11 \mathrm{mmHg}$, pulmonary artery wedge pressure of $10 \pm 4 \mathrm{mmHg}$ and pulmonary vascular resistance of $875 \pm 385 \mathrm{dyn} \cdot \mathrm{s} \cdot \mathrm{cm}^{-5}$. The median (range) time from diagnosis to study inclusion was 4.5 (2.5-7.3) years. All patients received medical therapy for pulmonary hypertension; the majority (64\%) received combination therapy with endothelin receptor antagonists and phosphodiesterase-5 inhibitors. These treatments were not changed during the course of the study.

Treatment with ferric maltol was well tolerated by the vast majority of patients. All but two patients completed the 12-week study period; the reasons for premature discontinuations were gastrointestinal side-effects ( $n=1$, related to study medication) and pneumonia ( $n=1$, unrelated to study medication). The only treatment-emergent adverse events occurring in two or more patients were diarrhoea $(n=3,14 \%$, including the aforementioned patient who discontinued study medication because of diarrhoea) and common cold $(n=2,9 \%)$. No deaths occurred during the main study period, but one patient died from right-sided heart failure during the 4 -week safety follow-up. This death was considered unrelated to study medication

The main results are shown in table 1: in the 20 patients who completed the 12 -week study period, haemoglobin (median $+2.9 \mathrm{~g} \cdot \mathrm{dL}^{-1}$, interquartile range $2.6-3.7 \mathrm{~g} \cdot \mathrm{dL}^{-1}$ ) and iron status significantly improved. In addition, we observed improvements in 6MWD and NT-proBNP. Echocardiography showed decreased RV dimensions and increased RV fractional area change, while tricuspid annular plane systolic excursion remained unchanged. Functional class improved in five patients, deteriorated in none and remained unchanged in the remaining patients. These changes were comparable to those observed previously with intravenous iron supplementation in patients with pulmonary hypertension and iron deficiency $[10,11]$.

\begin{tabular}{|c|c|c|c|}
\hline & Baseline & Week 12 & p-value \\
\hline Haemoglobin $\mathrm{g} \cdot \mathrm{dL}^{-1}$ & $10.7 \pm 0.9$ & $13.6 \pm 1.3$ & $<0.001^{\#}$ \\
\hline Iron $\mu \mathrm{mol} \cdot \mathrm{L}^{-1}$ & $5.4 \pm 2.0$ & $19.7 \pm 11.7$ & $<0.001^{\#}$ \\
\hline Transferrin saturation \% & $7.5 \pm 3.1$ & $31.7 \pm 19.6$ & $<0.001^{\#}$ \\
\hline Ferritin $\mu \mathrm{g} \cdot \mathrm{L}^{-1}$ & $13.1 \pm 6.7$ & $36.6 \pm 19.8$ & $<0.001^{\#}$ \\
\hline \multicolumn{4}{|l|}{ WHO FC } \\
\hline I & $0(0)$ & $0(0)$ & \multirow[t]{4}{*}{$0.091^{\pi}$} \\
\hline ॥ & $4(17)$ & $9(45)$ & \\
\hline III & $16(83)$ & $11(55)$ & \\
\hline IV & $0(0)$ & $0(0)$ & \\
\hline 6-min walk distance $\mathrm{m}$ & $331 \pm 147$ & $381 \pm 131$ & $0.004^{\#}$ \\
\hline NT-proBNP $n g \cdot L^{-1}$ & $496(254-902)$ & $298(160-484)$ & $0.003^{\#}$ \\
\hline Right atrial area $\mathrm{cm}^{2}$ & $23.7 \pm 8.4$ & $23.3 \pm 8.3$ & $0.070^{\#}$ \\
\hline Right ventricular diastolic diameter (four-chamber view) mm & $45.4 \pm 8.9$ & $42.3 \pm 8.4$ & $0.050^{\#}$ \\
\hline Right ventricular diastolic diameter (parasternal long axis) $\mathrm{mm}$ & $38.7 \pm 7.3$ & $35.0 \pm 6.3$ & $0.002^{\#}$ \\
\hline Right ventricular fractional area change \% & $30.5 \pm 12.8$ & $37.0 \pm 11.3$ & $0.005^{\#}$ \\
\hline TAPSE $\mathrm{mm}$ & $21.6 \pm 5.6$ & $21.3 \pm 5.6$ & $1.000^{\#}$ \\
\hline
\end{tabular}

Data are presented as mean \pm SD, $\mathrm{n}(\%)$ or median (interquartile range), unless otherwise stated. WHO FC: World Health Organization functional class; NT-proBNP: N-terminal prohormone of brain natriuretic peptide; TAPSE: tricuspid annular plane systolic excursion. ${ }^{\#}$ : nonparametric Wilcoxon test (paired); П: Chi-squared test. 
By design, our study had several limitations, including small sample size, open-label design, heterogeneous patient population and lack of a control group. The objective of the study was to generate preliminary data on the safety, tolerability and efficacy of ferric maltol in patients with pulmonary hypertension to set the stage for larger trials comparing this approach with intravenous iron supplementation. Despite the outlined limitations, we believe that the results of the study are encouraging and provide a clear signal to move forward.

In conclusion, in this exploratory open-label study in patients with pulmonary hypertension and iron deficiency anaemia, ferric maltol was well tolerated by the vast majority of patients and resulted in significant improvements in iron status and haemoglobin levels. These changes were accompanied by signs of improved right ventricular function and improved exercise capacity, supporting the notion that iron deficiency has detrimental effects in patients with pulmonary hypertension and that treating iron deficiency anaemia in these patients is important. Despite the limitations of a small, uncontrolled and unblinded study, our results suggest that oral iron supplementation with ferric maltol might become a safe, effective and convenient treatment option for patients with pulmonary hypertension and iron deficiency. Further studies are needed to compare oral and intravenous iron supplementation in this cohort of patients.

Karen M. Olsson ${ }^{1,2}$, Jan Fuge $\oplus^{1,2}$, Torben Brod ${ }^{1}$, Jan C. Kamp $\oplus^{1}$, Jan Schmitto ${ }^{3}$, Tibor Kempf ${ }^{4}$, Johann Bauersachs $\oplus^{4}$ and Marius M. Hoeper $\overbrace{}^{1,2}$

${ }^{1}$ Dept of Respiratory Medicine, Hannover Medical School, Hannover, Germany. ${ }^{2}$ German Centre of Lung Research (DZL), Germany. ${ }^{3}$ Dept of Cardiothoracic, Transplantation and Vascular Surgery, Hannover Medical School, Hannover, Germany. ${ }^{4}$ Dept of Cardiology and Angiology, Hannover Medical School, Hannover, Germany.

Correspondence: Karen M. Olsson, Dept of Respiratory Medicine, Hannover Medical School, 30623 Hannover, Germany. E-mail: olsson.karen@mh-hannover.de

Received: 10 March 2020 | Accepted after revision: 13 May 2020

Acknowledgements: We are indebted to the team of the Hannover Clinical Trial Center for providing logistic support throughout the study.

The study is registered at Clinicaltrials.gov with identifier number NCT03371173. Fully anonymised, individual patient data from this study can be obtained from J. Fuge (fuge.jan@mh-hannover.de).

Conflict of interest: K.M. Olsson reports personal fees for lectures and consultancy from Actelion, MSD and United Therapeutics, personal fees for consultancy from Accleron, outside the submitted work. J. Fuge has nothing to disclose T. Brod has nothing to disclose. J.C. Kamp has nothing to disclose. J. Schmitto reports personal fees for lectures and consultancy, and travel support from Medtronic, personal fees for lectures from Abbott, outside the submitted work. T. Kempf reports personal fees for lectures and consultancy from Vifor Pharma Ltd, personal fees for consultancy from Pharmacosmos A/S, personal fees for lectures from Novartis and AstraZeneca, outside the submitted work. J. Bauersachs reports personal fees for lectures and consultancy from Novartis, BMS, Pfizer, Daichii, Sankyo, Servier, MSD, Boehringer Ingelheim, AstraZeneca and Abbott, grants and personal fees for lectures and consultancy from Bayer, CVRx, Abiomed, Medtronic and Vifor, grants from Zoll, outside the submitted work. M.M. Hoeper reports personal fees for lectures and consultancy from Actelion, Bayer, MSD and Pfizer, personal fees for consultancy from Accleron, all outside the submitted work.

Support statement: This investigator-initiated study was funded by the Deutsche Forschungsgemeinschaft (KFO 331 TP1, HO 1599/2-1 and 1599/2-2; BA 1742/9-1 and BA 1742/9-2; SCHM 3076/1-1 and SCHM 3076/1-2; KE 1748/2-1 and KE 1748/2-2). Shield Therapeutics provided the study medication. Shield Therapeutics did not provide financial or logistic support to this study and was not involved in data analysis or writing of this manuscript. Funding information for this article has been deposited with the Crossref Funder Registry.

\section{References}

1 Rhodes CJ, Howard LS, Busbridge M, et al. Iron deficiency and raised hepcidin in idiopathic pulmonary arterial hypertension clinical prevalence, outcomes, and mechanistic insights. J Am Coll Cardiol 2011; 58: 300-309.

2 Rhodes CJ, Wharton J, Howard L, et al. Iron deficiency in pulmonary arterial hypertension: a potential therapeutic target. Eur Respir J 2011; 38: 1453-1460.

3 Ruiter G, Lankhorst S, Boonstra A, et al. Iron deficiency is common in idiopathic pulmonary arterial hypertension. Eur Respir J 2011; 37: 1386-1391.

4 von Haehling S, Ebner N, Evertz R, et al. Iron deficiency in heart failure: an overview. JACC Heart Fail 2019; 7: 36-46.

5 Ruiter G, Lanser IJ, de Man FS, et al. Iron deficiency in systemic sclerosis patients with and without pulmonary hypertension. Rheumatology 2014; 53: 285-292.

6 Galiè N, Humbert M, Vachiery JL, et al. 2015 ESC/ERS Guidelines for the diagnosis and treatment of pulmonary hypertension: The Joint Task Force for the Diagnosis and Treatment of Pulmonary Hypertension of the European Society of Cardiology (ESC) and the European Respiratory Society (ERS): Endorsed by: Association for European Paediatric and Congenital Cardiology (AEPC), International Society for Heart and Lung Transplantation (ISHLT). Eur Heart J 2016; 37: 67-119. 
7 Mulder MB, van den Hoek HL, Birnie E, et al. Comparison of hypersensitivity reactions of intravenous iron: iron isomaltoside-1000 (Monofer ${ }^{\star}$ ) versus ferric carboxy-maltose (Ferinject $\left.{ }^{\star}\right)$. A single center, cohort study. Br J Clin Pharmacol 2019; 85: 385-392.

8 Kelsey SM, Blake DR, Hider RC, et al. Absorption of ferric maltol, a novel ferric iron compound, in iron-deficient subjects. Clin Lab Haematol 1989; 11: 287-288.

9 Gasche C, Ahmad T, Tulassay Z, et al. Ferric maltol is effective in correcting iron deficiency anemia in patients with inflammatory bowel disease: results from a phase-3 clinical trial program. Inflamm Bowel Dis 2015; 21: 579-588.

10 Viethen T, Gerhardt F, Dumitrescu D, et al. Ferric carboxymaltose improves exercise capacity and quality of life in patients with pulmonary arterial hypertension and iron deficiency: a pilot study. Int J Cardiol 2014; 175: 233-239.

11 Ruiter G, Manders E, Happé CM, et al. Intravenous iron therapy in patients with idiopathic pulmonary arterial hypertension and iron deficiency. Pulm Circ 2015; 5: 466-472.

Copyright @eERS 2020.

This version is distributed under the terms of the Creative Commons Attribution Non-Commercial Licence 4.0. 